\title{
Update on the Treatment of Ataxia: Medication and Emerging Therapies
}

\author{
Susan L. Perlman ${ }^{1}$ (D) \\ Accepted: 28 September 2020 / Published online: 6 October 2020 \\ (C) The American Society for Experimental NeuroTherapeutics, Inc. 2020
}

\begin{abstract}
While rehabilitation therapies always help patients with ataxia, there are currently no FDA-approved treatments for ataxia. Medications are available to treat symptoms that may complicate an ataxic illness, e.g., tremor, myoclonus, dystonia, and rigidity, which are discussed elsewhere in this volume. Spasticity, pain, fatigue, depression, sleep disturbances, cognitive decline, and bowel and bladder dysfunction, if they occur, all have multiple available drugs and therapies for symptomatic use. There is also an extensive literature on off-label uses of various medications to improve imbalance. The pipeline of emerging therapies for symptomatic and possible disease-modifying management of ataxia gives hope that we will soon see the first of many FDAapproved drugs for ataxic illnesses.
\end{abstract}

Key Words Ataxia $\cdot$ rehabilitation $\cdot$ clinical trials $\cdot$ neurostimulation $\cdot$ gene therapy

\section{Introduction}

Ataxia, as a diagnosis, displays motor symptoms and signs of staggering gait, imbalance, incoordination, action tremor, slurred speech, trouble swallowing, disequilibrium/dizziness, nystagmus, and double vision, typically caused by cerebellar dysfunction on the basis of genetic, acquired, or degenerative causes [1-4].

While these symptoms can specifically come from the cerebellum itself, the disease process may also involve neural pathways outside the cerebellum, including intrinsic brainstem nuclei (oculomotor and bulbar dysfunction), spinal long tracts (spasticity, sensory changes), and supratentorial pathways (basal ganglia symptoms, frontal subcortical cognitive/mood changes [5]). Changes in special sensory pathways (optic, auditory), peripheral motor and sensory pathways, and autonomic pathways can accompany certain ataxic diagnoses. Involvement of any combination of these symptoms can lead to derangement of bowel and bladder function, altered sleep patterns, fatigue, pain, and mood disturbances.
Susan L. Perlman

sperlman@mednet.ucla.edu

1 Clinical Professor of Neurology, David Geffen School of Medicine at UCLA, Los Angeles, CA 90095, USA
"Pseudo-ataxia," when the cerebellum is normal, but gait, balance, and coordination are compromised by other factors, can be seen in conditions of muscle weakness, muscle stiffness (spasticity or rigidity), severe peripheral or posterior column sensory loss, peripheral vestibular dysfunction, visual problems, and orthopedic disease.

While the multifactorial presentation of an ataxic patient may seem overwhelmingly complex, utilizing any of the multiple available therapeutic interventions for the individual symptom areas can always help [6].

Stopping or slowing of disease progression, however, for any of the types of ataxia, depends on finding the specific causal mechanisms and the disease-modifying therapies that target them. There are as yet no disease-modifying therapies for genetic or degenerative cerebellar disorders. The treatable ataxias remain the acquired ones (vitamin deficiency, other metabolic disturbances, toxin exposure, medication side effects, autoimmune/paraneoplastic processes, neural infection or inflammation, structural lesions) [7].

\section{Rehabilitation}

Physical therapy, home exercise programs (including stretching, core strengthening, balance, and cardio), occupational therapy, speech and swallowing therapy, weighted vests [8], orthotics, assistive equipment assessment, home safety 
evaluations, and driving evaluations can all improve patient and family quality of life and safety, reduce falls, and help maintain patient independence $[9,10]$. Home Health organizations can provide these resources, as well as nursing and social work assistance, for the homebound.

\section{Non-invasive Neurostimulation}

A consensus panel agrees that both transcranial magnetic stimulation (single pulse or repetitive TMS) and transcranial direct current stimulation (anodal or cathodal tDCS) can effectively influence cerebellar motor functions, as well as visual tracking tasks, motor adaptation and learning, and cognitive and affective operations mediated by cerebro-cerebellar pathways [11]. They also agree that larger controlled trials will be needed for these non-invasive technologies to be approved for general symptomatic use, as well as to establish their safety and outcomes for long-term use.

\section{TMS}

The US Food and Drug Administration (FDA) permitted marketing of TMS as a treatment for major depression in 2008 and expanded the use to include TMS for treating pain associated with certain migraine headaches in 2013 and for obsessivecompulsive disorder in 2018. Over the past 20 years, multiple studies of small numbers of ataxic patients have explored the usefulness of TMS and rTMS for the development of biomarkers for early diagnosis and disease monitoring, as well as utilization of rTMS for treatment of ataxia [12]. A recent double-blind, randomized, sham-controlled trial of rTMS (5 days/week for 4 weeks) in twenty patients with genetically confirmed spinocerebellar ataxia showed, at 1 week and 1 month, improvements in the Scale for Assessment and Rating of Ataxia-SARA (stance subscale), as well as measures of postural sway with standing. Treatment did not influence the 9-hole peg test, Timed Up and Go test-TUG, or gait kinematics. Additional research was felt to be warranted to confirm longer-term benefits on standing postural control [13].

\section{DCS}

A double-blind, randomized, sham-controlled trial [14] and a similarly designed crossover trial [15] with cerebellar tDCS (5 days/week for 2 weeks) in twenty patients with neurodegenerative ataxia showed, at 1 and 3 months, a significant improvement in all performance scores (Scale for the Assessment and Rating of Ataxia-SARA, International Cooperative Ataxia Rating Scale-ICARS, 9-hole peg test, 8$\mathrm{m}$ walking time), effectively modulating cerebellar motor connectivity at short and long term.
Deep brain stimulation (DBS) has had preclinical and clinical work done on its influence on essential tremor pathways (cerebello-thalamo-cortical) that may also be involved in ataxia $[16,17]$, but one side effect of DBS is ataxia.

\section{Symptomatic Medication}

A Comprehensive Systematic Review Summary: Treatment of Cerebellar Motor Dysfunction and Ataxia (Report of the Guideline Development, Dissemination, and Implementation Subcommittee of the American Academy of Neurology) [18] found there to be class I evidence over 2-3 months to a year for the effectiveness of 4-aminopyridine $15 \mathrm{mg} /$ day in episodic ataxia type 2 and riluzole $50 \mathrm{mg}$ every $12 \mathrm{~h}$ in cerebellar ataxia and Friedreich's ataxia.

Multiple off-label agents (e.g., amantadine, buspirone, varenicline - discussed on the National Ataxia Foundation website-https://ataxia.org/what-is-ataxia/treatment) used over the past 40 years and reviewed by the committee had weaker, conflicting, or insufficient evidence of efficacy.

There are several symptomatic drugs in development for cerebellar ataxia, all directed at improving Purkinje cell function. Troriluzole, a pro-drug of riluzole, is in a phase 3 trial for spinocerebellar ataxia by Biohaven Pharmaceuticals, Inc. (ClinicalTrials.gov Identifier: NCT03701399); Cadent Therapeutics will be starting their phase 2 trial of CAD-1883 for spinocerebellar ataxia (ClinicalTrials.gov Identifier: NCT04301284); Takeda completed a phase 2 trial of TAK831 in Friedreich's ataxia (ClinicalTrials.gov Identifier: NCT03214588). Riluzole, a small-conductance calcium-activated potassium channel (SK channel) opener and glutamate transporter enhancer, mitigated deficits in a rat model of ataxia via reducing over-excitability of Purkinje neurons [19].

CAD-1883, a small molecule positive allosteric modulator of SK channels, may also regularize Purkinje cell firing.

TAK-831, a small molecule D-amino acid oxidase (DAO) inhibitor, leads to a measurable increase in D-serine levels in plasma, CSF, and cerebellum in rodents. D-Serine, a potent $\mathrm{N}$ methyl D-aspartate (NMDA)-type glutamate receptor coagonist and an agonist for the glutamate receptor subunit delta 2 , may lead to an improvement in cerebellar output in the form of motor learning and conditioning [20].

IntraBio Inc. is studying a new formulation (N-acetyl-Lleucine) of acetyl DL leucine used in France for 60 years for vertigo under the brand name Tanganil, which may regulate the membrane potential of cerebellar Purkinje cells and influence motor control and adaptive vestibular-cerebellar mechanisms (ClinicalTrials.gov Identifier: NCT03759678; ClinicalTrials.gov Identifier: NCT03759665).

Erydel has developed an intra-erythrocyte delivery technology, currently in testing for dexamethasone in ataxia telangiectasia (ClinicalTrials.gov Identifier: NCT02770807). 


\section{Vitamins, Supplements, and Complementary Medicine}

When nutrition or weight loss is a concern (with respect to calorie-protein or micronutrient deficiencies due to trouble swallowing or food intolerances), vitamins and dietary supplements are often recommended [21].

Neurodegenerative movement disorders with suspected mitochondrial dysfunction have had a number of published studies of anti-oxidant nutraceuticals felt to be mitochondrial enhancers (often shared from the anti-aging literature) [22]. Friedreich's ataxia is the most represented of the ataxic disorders, having had trials of vitamin $\mathrm{E}$, two vitamin $\mathrm{E}$ derivatives (Penwest Pharmaceuticals-A0001; Edison PharmceuticalsEPI-743, now acquired by PTC Therapeutics), coenzyme Q10, idebenone (Santhera Pharmaceuticals-a modified form of coenzyme Q10), acetyl-L-carnitine, resveratrol, and Cardero Therapeutics - Epicatechin, Ipsen-Egb761 a derivative of Gingko, Shire-VP-20629. With the exception of EPI743 [23], none have published statistically significant symptomatic benefits. An observational study of coenzyme Q10 use in spinocerebellar ataxia suggested better clinical outcomes for SCA1 and SCA3, but no change in clinical progression over 2 years [24].

Naturopathic, homeopathic, herbal, and other popular complementary therapies can certainly be tried under supervision and for a reasonable period of time, but it is important to research any possible side effects before allowing a patient to experiment with these approaches [25-27].

\section{Pipeline for Disease-Modifying Therapies}

Understanding of the mechanisms underlying neurodegeneration in cerebellar ataxia is a strong lead to the development of disease-modifying therapies [28]. This has been beautifully laid out in the pipeline for drug development for Friedreich's ataxia on the FARA (Friedreich's Ataxia Research Alliance) website (https://www.curefa.org/pipeline) [29]. The pipeline for spinocerebellar ataxia continues to expand [30].

\section{Neuroprotective}

The cascade of interacting molecular events that leads to selective nerve cell death in various neurodegenerative disorders is still incompletely understood, and the key steps that drive it are not identified in a way that could provide reliable targets for drug development $[31,32]$. Initiated by aging-related, genetic, and environmental factors and their influence on neuronal homeostasis (mitochondrial function, neuroinflammation), what follows is activation of pro-degenerative pathways (caspases and other "executioners") and ultimately death of the neuron (apoptosis, necroptosis, autophagy). But which step is the most important one to modify, in order to "put the brakes on" this process $[33,34]$ ?

\section{Anti-apoptotic/Anti-excitotoxicity}

ALS research has served as a model of aggressive investigation into neuroprotective agents [35], but after decades of trials there are only two FDA-approved drugs for ALS - the first being riluzole, which has only a modest effect on survival. Riluzole protects against excitotoxic motor neuron degeneration by interrupting glutamatergic transmission and lowering glutamate concentration, with effects on NMDA or AMPA receptors. European studies suggest it could have a similar effect in spinocerebellar degeneration [36].

\section{Anti-neuroinflammatory}

Inflammatory mediators released by the innate immune cells of the brain (microglia and astrocytes) can compromise the function and structure of neurons, playing important roles in the pathogenesis of neurodegenerative diseases [37, 38].

Minocycline has been studied preclinically [39] and in clinical trials as a potential modifier of neuroinflammation and caspase activity in neurodegenerative diseases.

Biohaven Pharmaceuticals, Inc., has begun a phase 3 study of Verdiperstat (a myeloperoxidase inhibitor) for multiple system atrophy (ClinicalTrials.gov Identifier: NCT03952806).

\section{Mitochondrial Enhancement}

Neurodegenerative movement disorders with suspected secondary mitochondrial dysfunction (Huntington's disease, spinocerebellar ataxia) [28] have had mitochondrial enhancement proposed as a neuroprotective strategy to reduce oxidative stress, sustain energy production, and delay nerve cell death. Candidate drugs targeting mitochondrial function in Friedreich's ataxia (phase 3 study of the Nrf2 activator omaveloxolone by Reata Pharmaceuticals, Inc.ClinicalTrials.gov Identifier: NCT02255435; phase 3 study of the deuterated polyunsaturated fatty acid RT001 by Retrotope, Inc. - ClinicalTrials.gov Identifier: NCT04102501) may have application to other neurodegenerative disorders with a mitochondrial component.

\section{Neural Replacement}

Stem cell therapies for neurologic disease have had suggested mechanisms that range from neural replacement to nerve 
growth factor enhancement to use as a delivery vehicle for other therapy [40-42]. A phase 1/2a open-label study of intravenous adipose-derived mesenchymal stem cell treatment in 6 patients with spinocerebellar ataxia type 3 (ClinicalTrials.gov Identifier: NCT01649687) demonstrated good safety and tolerability over 1 year with plans to conduct a larger, controlled trial in the future [43].

\section{Genetic and Epigenetic Approaches}

In Friedreich's ataxia, there are at least six groups working on AAV vector-based gene replacement therapies [44]. Management of the anti-capsid and anti-transgene neuroinflammatory response as it impacts safety and efficacy remains a challenge [45].

Anti-sense oligonucleotide (ASO) therapies are being investigated in the CAG triplet repeat spinocerebellar ataxias (SCA1, 2, 3, 6, 7), using the model being tested in HD (Hoffmann-La Roche - ClinicalTrials.gov Identifier: NCT03761849; Wave Life Sciences Ltd.-ClinicalTrials.gov Identifier: NCT03225833, ClinicalTrials.gov Identifier: NCT03225846; UniQure Biopharma B.V.-ClinicalTrials.gov Identifier: NCT04120493), for targeting the associated mRNA for destruction before the polyglutamine protein can be translated [46].

Gene editing with CRISPR/Cas9 as a way to remove the pathologically expanded triplet repeats (GAA in Friedreich's ataxia, CAG in spinocerebellar ataxia) is also being explored [47, 48].

Epigenetic approaches, to "make the abnormal gene behave like a normal gene," are being tested in Friedreich's ataxia with small molecules, ASOs, and artificial transcription factors that enable transcription to "read-through" the GAA expansion "and make protein anyway [49]." Micro RNAs have been looked at in spinocerebellar ataxia as an epigenetic way to modify mutant DNA transcription [50-53].

In genetic diseases where there is a reduction in a protein level (usually recessively inherited disorders), diseasemodifying therapies can include protein replacement or other ways to manipulate the metabolic pathway involved (e.g., in lysosomal storage diseases, the use of substrate reduction therapies; in other disorders, strategies to reduce breakdown of the deficient protein). In Friedreich's ataxia, there is a current phase 1 study of CTI-1601 for frataxin protein replacement by Chondrial Therapeutics, Inc.-ClinicalTrials.gov Identifier: NCT04176991.

\section{Looking Forward}

It is unlikely that there will be one "magic bullet" that will control all the processes above and be approved as "the cure" for ataxia. There will most likely be a "cocktail" of agents, some disease-specific and some ataxia-specific, that will ultimately turn the neurodegenerative cerebellar disorders into treatable diseases. But, in working with the ataxic patient and family, it is important to remember that there is always something you can do, even if it just educating, listening, and having the conversation.

Required Author Forms Disclosure forms provided by the authors are available with the online version of this article.

\section{References}

1. Brandsma R, Verschuuren-Bemelmans CC, Amrom D, Barisic N, Baxter P, Bertini E, et al. A clinical diagnostic algorithm for early onset cerebellar ataxia. Eur J Paediatr Neurol. 2019;23(5):692-706.

2. de Silva RN, Vallortigara J, Greenfield J, Hunt B, Giunti P, Hadjivassiliou M. Diagnosis and management of progressive ataxia in adults. Pract Neurol. 2019;19(3):196-207.

3. Soong BW, Morrison PJ. Spinocerebellar ataxias. Handb Clin Neurol. 2018;155:143-74.

4. Beaudin M, Matilla-Duenas A, Soong BW, Pedroso JL, Barsottini OG, Mitoma H, et al. The Classification of Autosomal Recessive Cerebellar Ataxias: a Consensus Statement from the Society for Research on the Cerebellum and Ataxias Task Force. Cerebellum. 2019;18(6):1098-125.

5. Schmahmann JD. The role of the cerebellum in cognition and emotion: personal reflections since 1982 on the dysmetria of thought hypothesis, and its historical evolution from theory to therapy. Neuropsychol Rev. 2010;20(3):236-60.

6. Stephen CD, Brizzi KT, Bouffard MA, Gomery P, Sullivan SL, Mello J, et al. The Comprehensive Management of Cerebellar Ataxia in Adults. Curr Treat Options Neurol. 2019;21(3):9.

7. Pedroso JL, Vale TC, Braga-Neto P, Dutra LA, Franca MC, Jr., Teive HAG, et al. Acute cerebellar ataxia: differential diagnosis and clinical approach. Arq Neuropsiquiatr. 2019;77(3):184-93.

8. Widener GL, Conley N, Whiteford S, Gee J, Harrell A, GibsonHorn $\mathrm{C}$, et al. Changes in standing stability with balance-based torso-weighting with cerebellar ataxia: A pilot study. Physiother Res Int. 2020;25(1):e1814.

9. Hartley H, Cassidy E, Bunn L, Kumar R, Pizer B, Lane S, et al. Exercise and Physical Therapy Interventions for Children with Ataxia: A Systematic Review. Cerebellum. 2019;18(5):951-68.

10. Howe TE, Rochester L, Neil F, Skelton DA, Ballinger C. Exercise for improving balance in older people. Cochrane Database Syst Rev. 2011(11):CD004963.

11. Grimaldi G, Argyropoulos GP, Boehringer A, Celnik P, Edwards MJ, Ferrucci R, et al. Non-invasive cerebellar stimulation-a consensus paper. Cerebellum. 2014;13(1):121-38.

12. Rodriguez-Labrada R, Velazquez-Perez L, Ziemann U. Transcranial magnetic stimulation in hereditary ataxias: Diagnostic utility, pathophysiological insight and treatment. Clin Neurophysiol. 2018;129(8):1688-98.

13. Manor B, Greenstein PE, Davila-Perez P, Wakefield S, Zhou J, Pascual-Leone A. Repetitive Transcranial Magnetic Stimulation in Spinocerebellar Ataxia: A Pilot Randomized Controlled Trial. Front Neurol. 2019;10:73.

14. Benussi A, Dell'Era V, Cotelli MS, Turla M, Casali C, Padovani A, et al. Long term clinical and neurophysiological effects of cerebellar transcranial direct current stimulation in patients with neurodegenerative ataxia. Brain Stimul. 2017;10(2):242-50. 
15. Benussi A, Dell'Era V, Cantoni V, Bonetta E, Grasso R, Manenti R, et al. Cerebello-spinal tDCS in ataxia: A randomized, double-blind, sham-controlled, crossover trial. Neurology. 2018;91(12):e1090-e101.

16. Miterko LN, Baker KB, Beckinghausen J, Bradnam LV, Cheng MY, Cooperrider J, et al. Consensus Paper: Experimental Neurostimulation of the Cerebellum. Cerebellum. 2019;18(6):1064-97.

17. Isobe T, Sato H, Goto T, Yako T, Yoshida K, Hashimoto T. LongTerm Suppression of Disabling Tremor by Thalamic Stimulation in a Patient with Spinocerebellar Ataxia Type 2. Stereotact Funct Neurosurg. 2019;97(4):241-3.

18. Zesiewicz TA, Wilmot G, Kuo SH, Perlman S, Greenstein PE, Ying $\mathrm{SH}$, et al. Comprehensive systematic review summary: Treatment of cerebellar motor dysfunction and ataxia: Report of the Guideline Development, Dissemination, and Implementation Subcommittee of the American Academy of Neurology. Neurology. 2018;90(10):464-71.

19. Janahmadi M, Goudarzi I, Kaffashian MR, Behzadi G, Fathollahi Y, Hajizadeh S. Co-treatment with riluzole, a neuroprotective drug, ameliorates the 3-acetylpyridine-induced neurotoxicity in cerebellar Purkinje neurones of rats: behavioural and electrophysiological evidence. Neurotoxicology. 2009;30(3):393-402.

20. Hopkins SC, Campbell UC, Heffernan ML, Spear KL, Jeggo RD, Spanswick DC, et al. Effects of D-amino acid oxidase inhibition on memory performance and long-term potentiation in vivo. Pharmacol Res Perspect. 2013;1(1):e00007.

21. Ronnefarth M, Hanisch N, Brandt AU, Mahler A, Endres M, Paul F, et al. Dysphagia Affecting Quality of Life in Cerebellar Ataxia-a Large Survey. Cerebellum. 2020.

22. Liu J, Wang LN. Mitochondrial enhancement for neurodegenerative movement disorders: a systematic review of trials involving creatine, coenzyme Q10, idebenone and mitoquinone. CNS Drugs. 2014;28(1):63-8.

23. Zesiewicz T, Salemi JL, Perlman S, Sullivan KL, Shaw JD, Huang Y, et al. Double-blind, randomized and controlled trial of EPI-743 in Friedreich's ataxia. Neurodegener Dis Manag. 2018;8(4):233-42.

24. Lo RY, Figueroa KP, Pulst SM, Lin CY, Perlman S, Wilmot G, et al. Coenzyme Q10 and spinocerebellar ataxias. Mov Disord. 2015;30(2):214-20.

25. Yu M, Bega D. A Review of the Clinical Evidence for Complementary and Alternative Medicine in Huntington's Disease. Tremor Other Hyperkinet Mov (N Y). 2019;9.

26. Posadzki P, Watson LK, Ernst E. Adverse effects of herbal medicines: an overview of systematic reviews. Clin Med (Lond). 2013;13(1):7-12.

27. Posadzki P, Watson L, Ernst E. Contamination and adulteration of herbal medicinal products (HMPs): an overview of systematic reviews. Eur J Clin Pharmacol. 2013;69(3):295-307.

28. Matilla-Duenas A, Ashizawa T, Brice A, Magri S, McFarland KN, Pandolfo M, et al. Consensus paper: pathological mechanisms underlying neurodegeneration in spinocerebellar ataxias. Cerebellum. 2014;13(2):269-302.

29. Gottesfeld JM. Molecular Mechanisms and Therapeutics for the GAA.TTC Expansion Disease Friedreich Ataxia. Neurotherapeutics. 2019;16(4):1032-49.

30. Kwei KT, Kuo SH. An Overview of the Current State and the Future of Ataxia Treatments. Neurol Clin. 2020;38(2):449-67.

31. Subramaniam S. Selective Neuronal Death in Neurodegenerative Diseases: The Ongoing Mystery. Yale J Biol Med. 2019;92(4):695705.

32. Andreone BJ, Larhammar M, Lewcock JW. Cell Death and Neurodegeneration. Cold Spring Harb Perspect Biol. 2020;12(2).

33. Prentice H, Modi JP, Wu JY. Mechanisms of Neuronal Protection against Excitotoxicity, Endoplasmic Reticulum Stress, and Mitochondrial Dysfunction in Stroke and Neurodegenerative Diseases. Oxid Med Cell Longev. 2015;2015:964518.
34. Bushart DD, Murphy GG, Shakkottai VG. Precision medicine in spinocerebellar ataxias: treatment based on common mechanisms of disease. Ann Transl Med. 2016;4(2):25.

35. Pandya RS, Zhu H, Li W, Bowser R, Friedlander RM, Wang X. Therapeutic neuroprotective agents for amyotrophic lateral sclerosis. Cell Mol Life Sci. 2013;70(24):4729-45.

36. Romano S, Coarelli G, Marcotulli C, Leonardi L, Piccolo F, Spadaro $\mathrm{M}$, et al. Riluzole in patients with hereditary cerebellar ataxia: a randomised, double-blind, placebo-controlled trial. Lancet Neurol. 2015;14(10):985-91.

37. Ising $\mathrm{C}$, Heneka MT. Functional and structural damage of neurons by innate immune mechanisms during neurodegeneration. Cell Death Dis. 2018;9(2):120.

38. Barake F, Soza A, Gonzalez A. Galectins in the brain: advances in neuroinflammation, neuroprotection and therapeutic opportunities. Curr Opin Neurol. 2020.

39. Kalonia H, Mishra J, Kumar A. Targeting neuro-inflammatory cytokines and oxidative stress by minocycline attenuates quinolinicacid-induced Huntington's disease-like symptoms in rats. Neurotox Res. 2012;22(4):310-20.

40. Cendelin J. Transplantation and Stem Cell Therapy for Cerebellar Degenerations. Cerebellum. 2016;15(1):48-50.

41. Akhlaghpasand M, Tizro M, Raoofi A, Meymand AZ, Farhadieh M, Khodagholi F, et al. Grafted human chorionic stem cells restore motor function and preclude cerebellar neurodegeneration in rat model of cerebellar ataxia. Metab Brain Dis. 2020.

42. Tajiri N, Staples M, Kaneko Y, Kim SU, Zesiewicz TA, Borlongan $\mathrm{CV}$. Autologous stem cell transplant with gene therapy for Friedreich ataxia. Med Hypotheses. 2014;83(3):296-8.

43. Tsai YA, Liu RS, Lirng JF, Yang BH, Chang CH, Wang YC, et al. Treatment of Spinocerebellar Ataxia With Mesenchymal Stem Cells: A Phase I/IIa Clinical Study. Cell Transplant. 2017;26(3):503-12.

44. Gerard C, Xiao X, Filali M, Coulombe Z, Arsenault M, Couet J, et al. An AAV9 coding for frataxin clearly improved the symptoms and prolonged the life of Friedreich ataxia mouse models. Mol Ther Methods Clin Dev. 2014;1:14044.

45. Perez BA, Shutterly A, Chan YK, Byrne BJ, Corti M. Management of Neuroinflammatory Responses to AAV-Mediated Gene Therapies for Neurodegenerative Diseases. Brain Sci. 2020;10(2).

46. Scoles DR, Minikel EV, Pulst SM. Antisense oligonucleotides: A primer. Neurol Genet. 2019;5(2):e323.

47. Ouellet DL, Cherif K, Rousseau J, Tremblay JP. Deletion of the GAA repeats from the human frataxin gene using the CRISPRCas9 system in YG8R-derived cells and mouse models of Friedreich ataxia. Gene Ther. 2017;24(5):265-74.

48. Ouyang S, Xie Y, Xiong Z, Yang Y, Xian Y, Ou Z, et al. CRISPR/ Cas9-Targeted Deletion of Polyglutamine in Spinocerebellar Ataxia Type 3-Derived Induced Pluripotent Stem Cells. Stem Cells Dev. 2018;27(11):756-70.

49. Gottesfeld JM. Milestones in transcription and chromatin published in the Journal of Biological Chemistry. J Biol Chem. 2019;294(5): 1652-60.

50. Martier R, Sogorb-Gonzalez M, Stricker-Shaver J, HubenerSchmid J, Keskin S, Klima J, et al. Development of an AAVBased MicroRNA Gene Therapy to Treat Machado-Joseph Disease. Mol Ther Methods Clin Dev. 2019;15:343-58.

51. Dong X, Cong S. The Emerging Role of microRNAs in Polyglutamine Diseases. Front Mol Neurosci. 2019;12:156.

52. Serrano M. Epigenetic cerebellar diseases. Handb Clin Neurol. 2018;155:227-44.

53. He F, Todd PK. Epigenetics in nucleotide repeat expansion disorders. Semin Neurol. 2011;31(5):470-83.

Publisher's Note Springer Nature remains neutral with regard to jurisdictional claims in published maps and institutional affiliations. 